\title{
A Correlation Study between Clinical Efficacy of Weifuchun on Precancerous Lesions of Gastric Cancer and Intestinal flora
}

\section{Yanqin Bian}

Shanghai University of Traditional Chinese Medicine

\section{Xi Chen}

Shanghai University of TCM: Shanghai University of Traditional Chinese Medicine

\section{Hongyan Cao}

Shanghai University of TCM-Integrated Hosipital

\section{Dong Xie}

Shanghai University of Traditional Chinese Medicine

\section{Meiping Zhu}

Shanghai University of Traditional Chinese Medicine

\section{Nong Yuan}

Shanghai University of Traditional Chinese Medicine Yueyang Hospital of Integrated Traditional Chinese Medicine and Western Medicine Department of Tui

Lu Lu

Shanghai University of Traditional Chinese Medicine institute of liver diseases

\section{Bingjie Lu}

Shanghai University of Traditional Chinese Medicine Shuguang Hospital

\section{Chao Wu}

Shanghai University of Traditional Chinese Medicine Institute of Liver Disease

\section{Nisma Lena Bahaji Azami}

Shanghai Traditional Chinese Medicine Shuguang Hospital

\section{Zheng Wang}

Shanghai University of Traditional Chinese Medicine Institute of Liver Disease

\section{Huijun Wang}

Shanghai Pharmaceuticals Holding Co Ltd

\section{Yeqing Zhang}

Shanghai Pharmaceuticals Holding Co Ltd

\section{Kun Li}

Shanghai Pharmaceuticals Holding Co Ltd

\section{Mingyu Sun ( $\nabla$ mysun248@hotmail.com )}

Shanghai University of Traditional Chinese Medicine Institute of Liver Disease

\section{Guan Ye}

Shanghai Pharmaceuticals Holding Co Ltd

\section{Research}


Keywords: precancerous lesions of gastric cancer, intestinal microbiota, Weifuchun, randomized controlled clinical trial Posted Date: June 7th, 2021

DOI: https://doi.org/10.21203/rs.3.rs-571877/v1

License: (c) (i) This work is licensed under a Creative Commons Attribution 4.0 International License. Read Full License 


\section{Abstract}

Backgroud

Weifuchun(WFC), a Chinese herbal prescription consisting of Red Ginseng, Isodon amethystoides and Fructus Aurantii, is commonly used in China to treat a variety of chronic stomach disorders. The aim of the paper is to determine the effect of WFC on intestinal microbiota changes in precancerous lesions of gastric cancer (PLGC) patients.

Methods

PLGC patients of H.pylori negative were randomly divided into two groups received either WFC tablets at a dose of $1.44 \mathrm{~g} 3$ times a day or vitacoenzyme tablets at a dose of $0.8 \mathrm{~g} 3$ times a day. All patients were treated for 6 months, consecutively. Gastroscopy and histopathology were used to assess the histopathological changes in the gastric tissues before and after treatment. 16S rRNA gene sequencing was carried out to assess the effects WFC on intestinal microbiota changes in PLGC patients. Receiver Operating Characteristics(ROC) analysis was used to assess the sensitivity and specificity of different intestinal microbiota in distinguishing between PLGC patients and healthy control.

Results

Gastroscopy and histopathological results indicated that WFC could improve the pathological condition of PLGC patients, especially in the atrophy or intestinal metaplasia. The results of $16 \mathrm{~S}$ rRNA gene sequencing indicated that WFC could regulate the microbial diversity, microbial composition, and abundance of the intestinal microbiota of PLGC patients. Following WFC treatment, the relative abundance of Parabacteroides was decreased in WFC group when compared with the control group. ROC analysis found that the Parabacteroides could effectively distinguish PLGC patients from healthy individuals with sensitivity of 0.79 and specificity of 0.8 .

\section{Conclusions}

WFC could slow down the progression of PLGC by regulating intestinal microbiota abundance.

Trial registration

NCT03814629. Name of registry: Randomized Clinical Trial: Weifuchun Treatment on Precancerous Lesions of Gastric Cancer. Registered 3 August 2018-Retrospectively registered, https://register.clinicaltrials.gov/ NCT03814629.

\section{Background}

Among all cancers, gastric cancer ranks fifth in terms of incidence and third in terms of mortality worldwide ${ }^{[1]}$. More than $70 \%$ of new gastric cancer cases are found in developing countries. It is reported that $42.6 \%$ of the global incidence and $45 \%$ of all gastric cancer-related deaths occur in China ${ }^{[2]}$, which is attributed to the low screening rate and diagnosis rate of early gastric cancer. Early detection of precancerous lesions of gastric cancer (PLGC) to halt their further development can effectively reduce the incidence of gastric cancer.

Recent research has established that gastric cancer is associated with bacterial dysbiosis within the stomach, especially Helicobacter pylori (H.pylori) in the stomach. There is increasingly compelling evidence that the microbiome can affect gastrointestinal carcinogenesis [3], especially gut microbiota, which plays a role in gastric cancer formation, development and response to treatment ${ }^{[4]}$. Recent advances in metagenomics and bioinformatics have provided new 
insights on the microbial ecology in gastric tumor. By using advanced sequencing technology, more intestinal flora involved in gastric cancer occurrence and cancer treatment can be found.

So far, there is no one drug proved to be effective on the PLGC excluding anti- H.pylori therapy. In China, vitacoenzyme tablet is approved for treatment atrophic gastritis and esophageal epithelial hyperplasia, which is a compound preparation from plant Soybean and main components of it are riboflavin and riboflavin derivative. As a positive drug, vitacoenzyme involved in several studies about chronic atrophic gastritis $(\mathrm{CAG})^{[5]}$ and gastric precancerous lesions $(\mathrm{GPL})^{[67]}$. Those studies found that vitacoenzyme had the limited effect of protect gastric mucosa against CAG.

Weifuchun (WFC) tablet is a well-known Chinese herbal drug, which is approved for market by China food and drug administration (CFDA) in 1982. WFC became a kind of the protected herbal drug varieties of CFDA in 1995. It is composed of three herbs, Renshen (Red Ginseng), Xiangchacai (Isodon amethystoides) and Zhike (Fructus Aurantii), and has the effects of strengthening the spleen and replenishing qi, promoting blood circulation and detoxification, eliminating gas and phlegm ${ }^{[8]}$. Currently, WFC has been widely used in the treatment of a variety of chronic stomach disorders including CAG and GPL ${ }^{[010]}$. Annual sales of WFC are 260 million US dollars in 2018. Modern studies showed that WFC could inhibit inflammation of H.pylori infected gastric epithelial cells by blocking NF-kappaB pathways ${ }^{[11]}$. Studies also demonstrated that WFC was antispasmodic and analgesic and its functions included regulating gastrointestinal motility ${ }^{[12]}$, inhibiting gastric acid secretion and protecting the gastric mucosa ${ }^{[1213]}$, and improving histological endoscopic findings and symptoms ${ }^{[10]}$ of PLGC.

However, there is not enough evidence in clinical trial regarding WFC ability to relieve PLGC and the mechanism of it is still unknown at present. More large scale randomized and control trials are needed to investigate WFC's effectiveness on PLGC. In this study, we evaluated WFC's effect on PLGC and assessed histopathological changes using a randomized and controlled trial. The stool samples of patients were collected to analyze the intestinal microbial abundance by high-throughput sequencing 16SrRNA. The results elucidate the probable mechanism of action of WFC in regulating intestinal microbial and treating atrophy and intestinal metaplasia(IM) to alleviate PLGC.

\section{Methods}

1. Quality and Quantity Analyses of Weifuchun

WFC tablet was kindly provided by Huqingyu-tang Pharmaceutical Co., Ltd. (Hangzhou, China). Quality and quantity analyses of the aqueous extract were performed with UPLC TOF-MS. HPLC-grade acetonitrile, methanol, and formic acid were purchased from Fisher Scientic (Santa Clara, USA). Naringin, ginsenoside Rb1, and oridonin were identified in WFC by UPLC TOF-MS. The following conditions were used to analyze naringin, ordionin, and ginsenoside Rb1: system, Acquity UPLC system (Waters, USA), which consists of a solvent degasser, a binary pump, an auto-sampler and a column oven; column, Acquity UPLC BEH C18 RP column (1.7 $\mu \mathrm{m}, 100 \mathrm{~mm} \times 2.1 \mathrm{~mm}$ i.d.; Waters, USA); mobile phase A, $0.1 \%$ formic acid in water; mobile phase $B, 100 \%$ acetonitrile; flow rate, $0.3 \mathrm{~mL} / \mathrm{min}$; wavelengths, $210 \mathrm{~nm}$ for ginsenoside Rb1, $254 \mathrm{~nm}$ for ordionin and $280 \mathrm{~nm}$ for naringin; injection volume, $10 \mu \mathrm{L}$; MS/MS detector, Acquity Synapt G2 Q-TOF tandem mass spectrometer connected to the UPLC system by an ESI interface and controlled by MassLynx version 4.1 (Waters, UK). Samples were analyzed in the positive model. Data were collected and analyzed by Waters MassLynx version 4.1.

\section{Trial Oversight}

This randomized and controlled trial was conducted in the outpatient clinics of Shuguang Hospital and Shanghai TCMIntegrated Hospital affiliated to Shanghai University of Traditional Chinese Medicine. All subjects (patients and health

Page $4 / 22$ 
volunteers) provided written informed consent before enrollment. The trial was approved by the institutional review board at Shuguang Hospital and was conducted in accordance with the provisions of the Declaration of Helsinki and the CONSORT guidelines. An independent data and safety monitoring board reviewed the progress of the trial.

The study protocol, which describes the study in more detail, can be found in the clinical trial registry (https://register.clinicaltrials.gov) with the identifier NCT03814629. The study was approved by the Ethics Committees of Shuguang Hospital affiliated to Shanghai University of Traditional Chinese Medicine (No. 2016-478-29-01). Recruitment and data collection occurred between October 2015 and September 2017. Patients with a previous histological diagnosis of CAG with or without IM/dysplasia were selected as potential subjects. Health volunteers were those never had stomach trouble and other serious disease. The trial was not registered until all the patients had been enrolled because registration was not mandated after the trial had started.

\section{Participants and Eligibility Criteria}

Only those who fulfilled the diagnosis of both CAG and IM or dysplasia, and H.pylori(-) were considered eligible subjects, male or female, between 18 and 70 years. Participants with H.pylori positive infection without radical treatment, peptic ulcer or severe dysplasia (suspected malignant transformation), severe systemic diseases such as cardiovascular and cerebrovascular disease, hepatic diseases, kidney or lung disease, or with other tumors, were excluded. Participants were excluded if they had an allergic constitution or allergies to any known ingredients in WFC. Finally, patients with other diseases interfering with the study or patients unwilling to undergo repeated endoscopy after treatment were also not included.

The TCM standard for diagnosing syndromes was worked out with reference to the standard for diagnosing the type of spleen and stomach weakness in the guidelines of diagnosing and treating CAG. Major symptoms are stomach pain or discomfort or stomach symptoms remission after warm or press operation. Minor symptoms include: (1) anorexia; (2) loose stools; (3) physical and mental fatigue; (4) shortness of breath and lazy speech; (5) stomach distention after eating; (6) belching; (7) chest distress; (8) stomach pain refused to press; and (9) light-colored tongue with small and wiry pulse. Patients with one the major symptom and two or more minor symptoms were diagnosed as suffering from the syndrome of spleen and stomach weakness.

\section{Trial Design and Treatment}

Before endoscopy, patients were randomly assigned in a 1:1 ratio to receive either WFC therapy or vitacoenzyme. Computer-generated randomization was performed in a blinded manner, with status concealed from all the patients and the primary physician, endoscopist, pathologist and statistician. After randomization, endoscopy was performed. The patients started the assigned trail medication within 1 week after endoscopy.

Each subject received either WFC tablets (1.44g) (Hangzhou huqingyutang pharmaceutical co. LTD. Hangzhou, China, lot number 16066129) or vitacoenzyme tablets (0.8g) (Beihai sunshine pharmaceutical co. LTD, Guangxi, China, lot number 102029), taken orally after meal 3 times a day for 6 months. Before randomization, $H$. pylori status was determined by rapid urea test (RUT) or by pathology. Positive subjects received standard eradication therapy. $\mathrm{H}$. pylori status was re-evaluated at the end of the 6th month. If required, the status would be re-evaluated by $13 \mathrm{C}$-urea breath test at 4 weeks after the cessation of therapy.

\section{Screening Measures}

The demographics of participants were collected, including age, gender, course of disease, and current and past gastric disease treatment. The histological diagnosis and grading was made according to the Consensus on Chronic Gastritis 
in China (Shanghai, 2012), the updated Sydney system ${ }^{[14]}$ and expert panel consensus.

\section{Histological scores}

Each gastric tissue was evaluated separately for the following items: chronic inflammation (Cl), acute inflammation (AI), atrophy, IM, and dysplasia. Atrophy was defined as loss of glands and graded as absent (0), mild (1), moderate (2), or severe (3). IM was graded as absent (0), mild(1), moderate (2), or severe (3) according to the proportion of the gastric mucosa replaced by the metaplastic issue. Presence and severity of dysplasia defined by atypical cytological and architectural derangement, subcategory of mild (1), moderate (2) and severe (3) grade adhered to the diagnosis for gastric neoplasia.

\section{High-throughput sequencing}

Total DNA was extracted using the QIAamp DNA Stool Mini Kit. All extractions yielding $>2 \mathrm{ng} / \mu$ of total DNA, as indicated by NanoDrop 2000 UV-Vis Spectrophotometer measuring. Each DNA sample was amplified for the V3 region of $16 \mathrm{~S}$ rRNA gene and libraries were sequenced in a single run of the lllumina MiSeq sequencing platform at NovelBio Biomedical technology Co.,LTD.

\section{Statistical analysis}

According the clinical report and previous research results ${ }^{[915]}$, we calculated the sample size of 35 patients in each group for the trial population. We allowed for a $15 \%$ initial dropout rate, and a further $10 \%$ loss to follow-up, resulting in the enrollment of 47 patients in each group. An interim analysis was not planned.

The statistical analyses were performed by using IBM SPSS Statistics 21.0. Data are expressed as mean \pm standard or median (range) for continuous variables, and frequencies (percentages) for categorical variables. Student's t-test or Mann-Whitney test or Chi square test was used to compare baselines including demographic data and basic evaluating variables. For comparison of variations from baseline to endpoint, paired t-test was performed on variables with normal distributions, and wilcoxon signed-ranks test on non-normal variables. For comparison microbial abundances between groups, the ANOVA was used. Chi-square test or Fisher's exact test was used for atrophy and intestinal metaplasia disappearance rate and symptom disappearance rate. All statistical tests were two-sided and assumed to be statistically significant at a level of $\mathrm{P}<0.05$.

\section{Results}

1. Naringin, ginsenoside Rb1, and oridonin Contents in the WFC Formula

Naringin, ginsenoside Rb1, and oridonin are the major constituents of aqueous extract of Radix Ginseng Rubra, Fructus aurantii, and Isodon amethystoides, respectively. The mass-to charge ratios (m/z) 581.1870, 1109.6107 in positive model for naringin and ginsenoside Rb1, and 363.1826 in negative model for oridonin, were observed in the peaks, confirming that they were protonated forms of naringin, ginsenoside Rb1, and oridonin, respectively. The details were shown in Fig.1.

\section{Baseline Characteristics for Participants}

Of the 87 patients who were screened, a total of 79 patients underwent randomization (Fig.2). Of these patients, 70 were included in the intention-to-treat population(36 in the WFC treatment group and 34 in the vitacoenzyme group) after the exclusion of 9 patients, including 3 who underwent additional surgery after endoscopic resection, and 4 who did not receive assigned treatment, and 2 who did not meet other eligibility requirements. Demographics (age, gender 
and course of disease), histopathology (histological score) and clinical symptom (aggregate score) were similar in the two groups (Table $\Downarrow$ ). We included 60 patients who had undergone gastroscopy at 6-month follow-up in the histologic analysis.

\section{Histology and clinical symptom.}

After treatment, the results of gastroscopy and histopathology were improved both in the WFC group and in the vitacoenzyme group (control group), especially in the aspect of atrophy and IM (Fig.3ABC). A large number of neat glands, less IM, reduced intercellular congestion edema and inflammatory cell infiltration was observed in histopathological findings in WFC group compared with the control group (Fig.3B). The total change value of pathology aggregate score in WFC group remarkably increased compared with the control group (Fig.3C). Patients with gastric mucosal atrophy or with IM in mild grade or moderate grade were the majority before treatment. But after treatment, patients with non-gastritis (normal) grade or mild grade in the WFC group were more than those in the control group, suggesting that WFC could improve atrophy and IM. The total effective rate for alleviating atrophy degree was $80 \%$ in the WFC group and $23.33 \%$ in the control group, respectively. And the total effective rate for alleviating IM degree was $73.33 \%$ in the WFC group and $26.67 \%$ in the control group, respectively (total effective rate of alleviating the atrophy

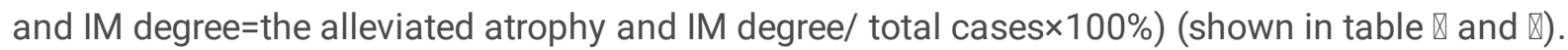

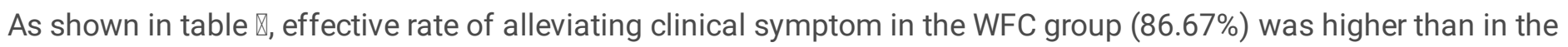
control group(23.33\%)(total effective rate for alleviating clinical symptom=(total clinical symptom score before treatment-total clinical symptom score after treatment)/before treatment $\times 100 \%$ ), indicating that WFC could dramatically improve clinical symptom.

\section{The taxonomic composition of intestinal microbiome.}

Sixty-six feces samples from all participants (28 samples from patients before and after treatment with WFC, 28 samples from patients before and after treatment with control drug, and 10 samples from healthy volunteers) were collected and sequenced the variable region V3 of the 16SrRNA using the Illumina MiSeq platform. A total 6,122,474 sequences ranging from 86,389 to 102,665 sequences per sample (mean=92,764.758; median=92,591) were obtained after quality control analyses. From these data, we identified a total of 62,453 operational taxonomic units (OTUs).

The intestinal microbiomes across all 66 samples included sequences that corresponded to 3 dominant $(>1.00 \%)$ Phyla: Firmicutes(52.74\%), Bacteroidetes (39.10\%) and Proteobacteria (6.44\%). These Phyla comprised 7 dominant $(>1.00 \%)$ class and 19 dominant $(>1.00 \%)$ genera. Top 6 dominant $(>3.00 \%)$ genera were Bacteroides $(24.51 \%)$, Lachnospiraceae(unclassified)(15.87\%), Faecalibacterium(6.25\%), Prevotella 9(6.07\%), Lachnoclostridium(3.37\%), and Parabacteroides(3.00\%). The mean relative proportion of dominant ( $>1 \%)$ phyla, class and genera in the five groups were shown in Fig 4. All these genera are commonly found in the feces of individuals with and without PLGC, although in different proportions ${ }^{[16-20]}$.

A variable number of OTUs from these 19 dominant (>1.00\%) genera were included in the core microbiome, which potentially comprises the stable and consistent members and association from the whole community ${ }^{[2122]}$. The least stringent definition of the core (presence in at least $50 \%$ of the samples) identified 127 OTUs of commensal and pathogenic bacteria; while a more stringent definition (presence in at least 95\% of the samples) included 8 OTUs of the following genera: Bacteroides,Lachnospiraceae(unclassified), Faecalibacterium, Lachnoclostridium, Parabacteroides, Streptococcus, Escherichia-Shigella, and Lachnospiraceae. Pathogenic representatives from Bacteroides,

Lachnospiracea, Faecalibacterium, Lachnoclostridium and Streptococcus genera have been consistently associated to gastric cancer ${ }^{[23-26]}$. Unlike the above 5 dominant genera, Parabacteroides could be more related to the gastric and 
intestinal disease such as dyspepsia[27], and Escherichia-Shigella andLachnospiraceae NK4A136 group were involved with gastrointestinal inflammation and immunity ${ }^{[2829]}$.

\section{Univariate, Receiver Operating Characteristics (ROC) Curve}

As shown in Fig.5, microbial abundances of all the eight dominant bacterial genera was compared between various groups based on the ANOVA, and between WFCB vs WFCA and ConB vs ConA by the paried t-test. All candidates except for Parabacteroides had no significantly differences among groups. Compared with Health group, the relative abundance of Parabacteroides was significantly higher in WFCB group. On the contrary, abundance of Parabacteroides declined observably in WFCA group. This decrease was not observed in the control group. The arithmetic mean \pm standard deviation of the relative abundance (raw count number reads) were 0.76 $\pm 0.76(14729.04 \pm 14767.28)$ for Health group, 1.86 \pm 1.01 (35775.48 \pm 20532.81$)$ for WFCB group and 0.98 $\pm 0.79(19062.95 \pm 15363.4)$ for WFCA group. 8 candidate genera were selected to analyze ability to assess PLGC using ROC curve. The results were shown in the Fig. 6 and in Table $\otimes$. The area under the curve (AUC) was $>0.7$ and $p$ value $<0.05$ only for Parabacteroides. The other 7 candidate genera were all $<0.7$ in AUC, suggesting that Parabacteroides may be a significant candidate to assess whether PLGC development or not and therapeutic effect of WFC on PLGC.

\section{Discussion}

Gastric cancer develops through a multistep process triggered by H.pylori and progression from superficial gastritis to atrophic gastritis, IM, and dysplasia ${ }^{[30]}$. Atrophy, IM or dysplasia is considered as PLGC and require accurate surveillance programs. PLGC belong to the "stomach distension" and "epigastralgia" category in traditional Chinese medicine. WFC is a clinical effective prescription for body discomfort including distension and fullness of the stomach, belching and poor appetite, constipation or diarrhea, lassitude and weakness, dizziness and emaciation, sallow complexion. In this study, the result showed that WFC could significantly improve gastric mucosa pathology especially atrophy and IM, and clinical symptoms.

The etiology of H.pylori-positive has been well described over the past few decades ${ }^{[31]}$, but H.pylori eradication cannot completely eliminate the recurrence risk of gastric cancer ${ }^{[32]}$, suggesting there is another factor affecting the progression of gastric cancer. It has been reported that the alternations of fecal microbiota involved in the process of H.pylori-related gastric lesion progression ${ }^{[33]}$. Resident microbes can induce inflammation, leading to cell proliferation and altered stem cell dynamics, which can lead to alternations in DNA integrity and immune regulation and promote carcinogenesis $^{[34]}$. In this study, we observed gut microbes alternation in PLGC population with H.pylori negative and the effect of WFC on this population. The result found that 8 kinds of microbes may dominate bacterial community of PLGC in genera level. Further analysis found that only the abundance of Parabacteroides was significantly different in Health group vs WFCB group and in WFCB group vs WFCA group. AUC was $>0.7$ for Parabacteroides in ROC analysis which could effectively distinguish PLGC patients from healthy individuals, with sensitivity of 0.79 and specificity of 0.8 , suggesting the importance of Parabacteroides in PLGC occurrence.

Parabacteroides genera belong to the Bacteroidetes phyla and the Porphyromonadaceae family. Among the gut Parabacteroides, Parabacteroides Distasonis (P. distasonis) is defined as one of the 18 core members in the gut microbiota of humans and thought to have important physiological functions in hosts ${ }^{[35]}$. Results from animal studies proved the protective role of $\mathrm{P}$. distasonis in colonic tumorigenesis and maintenance of intestinal epithelial barrier in AOM-treated mice ${ }^{[36]}$. A study from 736 American Gut Project sample found that the abundance of P. distasonis is relatively lower in patients with obesity, inflammatory bowel diseases, nonalcoholic fatty liver, and multiple sclerosis ${ }^{[37-}$ 
39]. Seemingly, the increase in relative abundance of Parabacteroides genera correlates with the absence of inflammation in the gut.

Nevertheless, recent evidence from in vivo and vitro confirmed that P.distasonis possessed a strong ability to transform primary bile acids into secondary bile acids and enhancing the level of succinate in the gut ${ }^{[2040]}$. The gut microbiota and the bile acid pool played pivotal roles in maintaining intestinal homeostasis. Interplay between bile acid and the gut microbiota promoted gastrointestinal carcinogenesis ${ }^{[41]}$. It was also reported that bacterial metabolites, including secondary bile acid, had the potential to cause direct DNA damage or to provoke inflammation, which in turn promoted carcinogenesis ${ }^{[42]}$. Bile acids could promote gastric IM by upregulating CDX2 and MUC2 expression via the FXR/NF-kB signaling pathway ${ }^{[43]}$. Succinate, the intermediates of the mitochondrial pathway known as the Krebs cycle, had extensive evidence for "non-metabolic" signaling functions or metabolic reprogramming leading to altered immune cell and transformed cell function in the initiation of carcinogenesis ${ }^{[4445]}$. Additionally, Parabacteroides in the gut could use type $\mathrm{VI}$ secretion systems(T6SSs) to antagonize symbiotic gut E. coli, facilitating colonization and cancer progression ${ }^{[46]}$. To sum up, Parabacteroides are multifunctional bacteria in the human gut and have the potential capacity to promote gastric carcinogenesis. In our study, the relative abundance of Parabacteroides in PLGC group was significantly high before treatment with WFC. In contrast, a decreased abundance of Parabacteroides was observed in PLGC group after treatment with WFC. The effect of WFC on gut Parabacteroides corresponded with the results of gastric histology in PLGC.

Accumulating data suggested that gut microbiota had a role in the etiology of several types of cancer, including gastric cancer. However, data about intestinal microbiota correlation with PLGC were not enough. Further studies on the alteration of intestinal flora in PLGC development are needed. The strength of our study is that both results from endoscopy and histology confirmed the curative effect of WFC on PLGC, which may be because of the abundance of gut microbiota redistribution, specially decreasing Parabacteroides. The study had few limitations including the limited sample size especially for collected feces for intestinal flora. Larger sample sizes are needed in order to confirm the role of Parabacteroides in the development of PLGC. Lastly, more detailed design illuminate causal inferences.

\section{Conclusions}

This study suggested WFC slow down PLGC, which could be related with Parabacteroides abundance variation. The results will help understand the effects of WFC on PLGC and provide a treatment method for PLGC.

\section{Abbreviations}

PLGC: precancerous lesions of gastric cancer; WFC: Weifuchun; CAG: chronic atrophic gastritis; GPL: gastric precancerous lesions; IM: intestinal metaplasia; Cl: chronic inflammation; Al: acute inflammation; CFDA: China food and drug administration; Health: healthy volunteers; WFCB: patients before treatment with WFC; WFCA: patients after treatment with WFC; ConB: patients before treatment with control drug; ConA: patients after treatment with control drug; OTUs: operational taxonomic units.

\section{Declarations}

\section{Ethics approval and consent to participate.}

The study was approved by the Ethics Committees of Shuguang Hospital affiliated to Shanghai University of Traditional Chinese Medicine (No. 2016-478-29-01) and performed in accordance with the Declaration of Helsinki. All 
patients were fully informed of the study and informed consent were obtained from all patients.

\section{Consent for publication.}

Not applicable.

\section{Availability of data and material.}

The datasets used and/or analyzed during the current study are available from the corresponding authors on reasonable request.

\section{Competing interests.}

The authors declare that they have no competing interests.

\section{Funding.}

This work was supported by the major Project of Shanghai Municipal Science and Technology Commission(No.15DZ1900104, 19401972300); The fourth batch of outstanding TCM talents of the State Administration of Traditional Chinese Medicine(2017-124); Innovation course of Postgraduate students in Shanghai University of Traditional Chinese Medicine(2017); Outstanding TCM talents of Shanghai University of Traditional Chinese Medicine(2020); Shanghai Key Laboratory of Traditional Chinese Clinical Medicine, Key Disciplines of Liver and Gall Bladder Diseases and Key Laboratory of Chronic Deficiency Liver Disease of State Administration of Traditional Chinese Medicine of the People's Republic of China. The study authors were independent of the funder. The funding sources had no involvement in the study.

\section{Authors' contributions.}

YQB, XC, GY and MYS conceived and designed the clinical trial; YQB and XC wrote the paper; MYS, MPZ and NY recruited patients; XC, HYC, DX, BJL, LL and CW collected the samples; YQB, XC, HYC and ZW analyzed clinical data; HJW and YQZ finished the drug quality and quantity analyses; NLBA helped proofread the manuscript; GY and MYS critically revised the paper for important intellectual content and gave final approval for publication of the paper. All authors read and approved the final manuscript.

Acknowledgements. We gratefully acknowledge Dr. Zhang and Dr. Guo from NovelBio Biomedical technology Co.,LTD, who provided us much technical help.

\section{References}

1. Torre LA, Bray F, Siegel RL, et al.. Global cancer statistics, 2012. CA Cancer J Clin 2015;65(2):87-108.

2. Wang W, Sun Z, Deng JY, et al.. A novel nomogram individually predicting disease-specific survival after D2 gastrectomy for advanced gastric cancer. Cancer Commun (Lond) 2018;38(1):23.

3. Chen J, Domingue JC, Sears CL. Microbiota dysbiosis in select human cancers: Evidence of association and causality. Semin Immunol 2017;32:25-34.

4. Wong SH, Kwong T, Wu CY, et al.. Clinical applications of gut microbiota in cancer biology. Semin Cancer Biol 2019;55:28-36.

5. Zhang J, Wang $\mathrm{H}$. Morroniside protects against chronic atrophic gastritis in rat via inhibiting inflammation and apoptosis. Am J Transl Res 2019;11(9):6016-6023. 
6. Peng L, Xie YF, Wang CG, et al.. Moxibustion alleviates gastric precancerous lesions in rats by promoting cell apoptosis and inhibiting proliferation-related oncogenes. Afr J Tradit Complement Altern Med 2017;14(2):148-160.

7. Zeng JH, Pan HF, Liu YZ, et al.. Effects of Weipixiao (미) on Wnt pathway-associated proteins in gastric mucosal epithelial cells from rats with gastric precancerous lesions. Chin J Integr Med 2016;22(4):267-75.

8. Chen X, Zhao YH, Zhang YQ, Ye G, Sun MY. Clinical applications and modern research progress of Weifuchun. Jiangxi Traditional Chinese Medicine 2016: 47:77-80 (In Chinese).

9. Li HZ, Wang H, Wang GQ, et al.. Treatment of gastric precancerous lesions with Weiansan. World J Gastroenterol 2006;12(33):5389-92.

10. Li Y, Xu JK, Uu XR. [Clinical and pathological study of weiyan serial recipes in the treatment of gastric precancerous lesions]. Zhongguo Zhong Xi Yi Jie He Za Zhi 2011;31(12):1635-8.

11. Huang $X$, Lu B, Zhang S, et al.. [Effect of Weifuchun on inhibiting inflammation of Helicobacter pylori-infected GES1 cells and NF-kappaB signaling pathway]. Zhongguo Zhong Xi Yi Jie He Za Zhi 2014;34(4):450-4.

12. Chen W, Chen WJ, Yang MH. Effect of Wei Fuchun table on gastrointestinal function in functional dyspesia rats. Chinese Journal of Modern Application Pharmacy 2019; 36:829-832. (In Chinese)

13. Xu HB, Chen QE, Chen CJ. Effect of matrine combined with Weifuchen tablets on gastric acid secrection in patients with chronic atrophic gastritis. World Chinese Journal of Digestology. 2017; 25:2139-43 (In Chinese)

14. Dixon MF, Genta RM, Yardley JH, et al.. Classification and grading of gastritis. The updated Sydney System. International Workshop on the Histopathology of Gastritis, Houston 1994. Am J Surg Pathol 1996;20(10):1161-81.

15. Deng X, Liu ZW, Wu FS, et al.. A clinical study of weining granules in the treatment of gastric precancerous lesions. J Tradit Chin Med 2012;32(2):164-72.

16. Sitkin S, Pokrotnieks J. Gut microbiota as a host defender and a foe: The 2 faces of commensal bacteroides thetaiotaomicron in inflammatory bowel disease. Inflamm Bowel Dis 2019;25(6):e71.

17. Lopez-Siles M, Duncan SH, Garcia-Gil LJ, et al.. Faecalibacterium prausnitzii: From microbiology to diagnostics and prognostics. Isme J 2017;11(4):841-852.

18. Ley RE. Gut microbiota in 2015: Prevotella in the gut: Choose carefully. Nat Rev Gastroenterol Hepatol 2016;13(2):69-70.

19. Youssef O, Lahti L, Kokkola A, Karla T, Tikkanen M, Ehsan H, et al.. Stool microbiota composition differs in patients with stomach, colon, and rectal neoplasms. Dig Dis Sci 2018;63(11):2950-58.

20. Wang K, Liao M, Zhou N, Bao L, Ma K, Zheng Z, et al.. Parabacteroides distasonis Alleviates Obesity and Metabolic Dysfunctions via Production of Succinate and Secondary Bile Acids. Cell Rep 2019;26(1):222-35.

21. Bäckhed F, Fraser CM, Ringel Y, Sanders ME, Sartor RB, Sherman PM, et al.. Defining a healthy human gut microbiome: Current concepts, future directions, and clinical applications. Cell Host Microbe 2012;12(5):611-22.

22. Shade A, Handelsman J. Beyond the Venn diagram: The hunt for a core microbiome. Environ Microbiol 2012;14(1):4-12.

23. Liu X, Shao L, Liu X, Ji F, Mei Y, Cheng Y, et al.. Alterations of gastric mucosal microbiota across different stomach microhabitats in a cohort of 276 patients with gastric cancer. Ebiomedicine 2019;40:336-48.

24. He C, Peng C, Wang H, Ouyang Y, Zhu Z, Shu X, et al.. The eradication of Helicobacter pylori restores rather than disturbs the gastrointestinal microbiota in asymptomatic young adults. Helicobacter 2019;24(4):e12590.

25. Gantuya B, El-Serag HB, Matsumoto T, Ajami NJ, Oyuntsetseg K, Azzaya D, et al.. Gastric microbiota in helicobacter pylori-Negative and -Positive gastritis among high incidence of gastric cancer area. Cancers (Basel) 2019;11(4).

26. Qi YF, Sun JN, Ren LF, Cao XL, Dong JH, Tao K, et al.. Intestinal microbiota is altered in patients with gastric cancer from shanxi province, china. Dig Dis Sci 2019;64(5):1193-203.

Page $11 / 22$ 
27. Gao B, Wang R, Peng Y, Li X. Effects of a homogeneous polysaccharide from Sijunzi decoction on human intestinal microbes and short chain fatty acids in vitro. J Ethnopharmacol 2018;224:465-73.

28. Li M, Bai Y, Zhou J, Huang W, Yan J, Tao J, et al.. Core fucosylation of maternal milk N-Glycan evokes b cell activation by selectively promoting the I-Fucose metabolism of gut bifidobacterium spp. And lactobacillus spp. Mbio 2019;10(2).

29. Bashir M, Prietl B, Tauschmann M, Mautner SI, Kump PK, Treiber G, et al.. Effects of high doses of vitamin D3 on mucosa-associated gut microbiome vary between regions of the human gastrointestinal tract. Eur $\mathrm{J}$ Nutr 2016;55(4):1479-89.

30. Nardone G, Rocco A, Malfertheiner P. Review article: Helicobacter pylori and molecular events in precancerous gastric lesions. Aliment Pharmacol Ther 2004;20(3):261-70.

31. Cadamuro Cadamuro AC, Rossi AF, Maniezzo NM, Silva AE. Helicobacter pylori infection: Host immune response, implications on gene expression and microRNAs. World J Gastroenterol 2014;20(6):1424-37.

32. 32. Choi IJ, Kook MC, Kim YI, Cho SJ, Lee JY, Kim CG, et al.. Helicobacter pylori therapy for the prevention of metachronous gastric cancer. N Engl J Med 2018;378(12):1085-95.

33. Gao JJ, Zhang Y, Gerhard M, Mejias-Luque R, Zhang L, Vieth M, et al.. Association between gut microbiota and helicobacter pylori-Related gastric lesions in a High-Risk population of gastric cancer. Front Cell Infect Microbiol 2018;8:202.

34. Wroblewski LE, Peek RJ, Coburn LA. The role of the microbiome in gastrointestinal cancer. Gastroenterol Clin North Am 2016;45(3):543-56.

35. Falony G, Joossens M, Vieira-Silva S, Wang J, Darzi Y, Faust K, et al.. Population-level analysis of gut microbiome variation. Science 2016;352(6285):560-64.

36. Koh GY, Kane AV, Wu X, Crott JW. Parabacteroides distasonis attenuates tumorigenesis, modulates inflammatory markers and promotes intestinal barrier integrity in azoxymethane-treated A/J mice. Carcinogenesis 2020;41(7):909-17.

37. 37. Cekanaviciute E, Yoo BB, Runia TF, Debelius JW, Singh S, Nelson CA, et al.. Gut bacteria from multiple sclerosis patients modulate human T cells and exacerbate symptoms in mouse models. Proc Natl Acad Sci U S A 2017;114(40):10713-18.

38. Del CF, Nobili V, Vernocchi P, Russo A, De Stefanis C, Gnani D, et al.. Gut microbiota profiling of pediatric nonalcoholic fatty liver disease and obese patients unveiled by an integrated meta-omics-based approach. Hepatology 2017;65(2):451-64.

39. Verdam FJ, Fuentes S, de Jonge C, Zoetendal EG, Erbil R, Greve JW, et al.. Human intestinal microbiota composition is associated with local and systemic inflammation in obesity. Obesity (Silver Spring) 2013;21(12):E607-15.

40. Ridlon JM, Devendran S, Alves JM, Doden H, Wolf PG, Pereira GV, et al.. The 'in vivo lifestyle' of bile acid 7adehydroxylating bacteria: Comparative genomics, metatranscriptomic, and bile acid metabolomics analysis of a defined microbial community in gnotobiotic mice. Gut Microbes 2020;11(3):381-404.

41. Wang S, Dong W, Liu L, Xu M, Wang Y, Liu T, et al.. Interplay between bile acids and the gut microbiota promotes intestinal carcinogenesis. Mol Carcinog 2019;58(7):1155-67.

42. Louis P, Hold GL, Flint HJ. The gut microbiota, bacterial metabolites and colorectal cancer. Nat Rev Microbiol 2014;12(10):661-72.

43. Yu JH, Zheng JB, Qi J, Yang K, Wu YH, Wang K, et al.. Bile acids promote gastric intestinal metaplasia by upregulating CDX2 and MUC2 expression via the FXR/NF-KB signalling pathway. Int J Oncol 2019;54(3):879-92. 
44. Ryan DG, Murphy MP, Frezza C, Prag HA, Chouchani ET, O'Neill LA, et al.. Coupling Krebs cycle metabolites to signalling in immunity and cancer. Nat Metab 2019;1:16-33.

45. Sajnani K, Islam F, Smith RA, Gopalan V, Lam AK. Genetic alterations in Krebs cycle and its impact on cancer pathogenesis. Biochimie 2017;135:164-72.

46. Coyne MJ, Comstock LE. Type VI secretion systems and the gut microbiota. Microbiol Spectr 2019;7(2).

\section{Tables}

Table \ Baseline Characteristics for Participants $(n=60)$ 


\begin{tabular}{|c|c|c|c|c|c|c|c|}
\hline & & $\begin{array}{l}\text { WFC } \\
(n=30)\end{array}$ & $\begin{array}{l}\text { Control } \\
(n=30)\end{array}$ & $\begin{array}{l}\text { Total } \\
(n=60)\end{array}$ & $x^{2}$ & $t$ & $P$ \\
\hline \multicolumn{2}{|c|}{$\mathrm{Age}^{\mathrm{a}}$} & $50.37(12.50)$ & $53.90(10.61)$ & $52.13(11.53)$ & & 1.2 & 0.243 \\
\hline \multirow[t]{2}{*}{ Sex } & Male & 16(53.33) & $15(50.00)$ & $31(51.67)$ & \multirow[t]{2}{*}{0.268} & & \multirow[t]{2}{*}{0.796} \\
\hline & Female & 14(46.67) & $15(50.00)$ & $29(48.33)$ & & & \\
\hline \multicolumn{2}{|c|}{ Course of disease ${ }^{a}$} & $5.57(4.94)$ & $5.87(5.10)$ & $5.72(4.93)$ & & 0.2 & 0.818 \\
\hline \multicolumn{8}{|c|}{ Pathology } \\
\hline & \multicolumn{7}{|l|}{ Chronic Gastritis $^{b}$} \\
\hline & Mild & $7(23.33)$ & 10(33.33) & 17(28.33) & & & \\
\hline & Moderate & $20(66.67)$ & 19(63.33) & $39(65.00)$ & & & \\
\hline & Severe & $2(6.67)$ & $1(3.33)$ & $3(5.00)$ & 1.888 & & 0.596 \\
\hline & \multicolumn{7}{|l|}{ Active Gastritis ${ }^{b}$} \\
\hline & Mild & $11(36.67)$ & $7(23.33)$ & $18(30.00)$ & & & \\
\hline & Moderate & $3(10.00)$ & $2(6.67)$ & $5(8.33)$ & & & \\
\hline & Severe & $0(0.00)$ & $0(0.00)$ & $0(0.00)$ & 1.765 & & 0.414 \\
\hline & \multicolumn{7}{|l|}{ Atrophy ${ }^{b}$} \\
\hline & Mild & $14(46.67)$ & 19(63.33) & $33(55)$ & & & \\
\hline & Moderate & $12(40)$ & 10(33.33) & $22(36.67)$ & & & \\
\hline & Severe & $4(13.33)$ & $1(3.33)$ & $5(8.33)$ & 3.000 & & 0.254 \\
\hline & \multicolumn{7}{|l|}{ Intestinal metaplasiab } \\
\hline & Mild & $16(53.33)$ & $21(70.00)$ & $37(61.67)$ & & & \\
\hline & Moderate & 10(33.33) & $7(23.33)$ & $17(28.33)$ & & & \\
\hline & Severe & $3(10.00)$ & $2(6.67)$ & $5(8.33)$ & 2.739 & & 0.493 \\
\hline & \multicolumn{7}{|l|}{ Dysplasia ${ }^{b}$} \\
\hline & Mild & $6(20.00)$ & $4(13.33)$ & 10(16.67) & 2.632 & & 0.235 \\
\hline & Moderate & $0(0.00)$ & $0(0.00)$ & $0(0.00)$ & & & \\
\hline & Severe & $0(0.00)$ & $0(0.00)$ & $0(0.00)$ & & & \\
\hline & Aggregate score ${ }^{c}$ & $5.70(2.00)$ & $4.97(1.63)$ & & & 1.6 & 0.125 \\
\hline \multicolumn{8}{|c|}{ Clinical Syptoms } \\
\hline & Aggregate score ${ }^{c}$ & $15.32(5.71)$ & & $15.92(5.43)$ & & 3.5 & 0.929 \\
\hline
\end{tabular}

1. Years, mean(SD); 
2. The number of cases meeting the diagnosis were counted and showed as mean (SD); absent cases were included when $P$ value were calculated through two-sided $X^{2}$ test.

3. Aggregate score represented sum of all grade score including absent, mild, moderate and severe; absent, 0; mild, 1; moderate, 2; severe, 3.

Table \. Comparison of Gastric Mucosal Atrophy Level

\begin{tabular}{|c|c|c|c|c|c|c|c|c|c|c|c|}
\hline \multirow[t]{2}{*}{ Group } & & \multirow[t]{2}{*}{$n$} & \multicolumn{4}{|c|}{ Atrophy Level (n) } & \multicolumn{4}{|c|}{$\begin{array}{l}\text { Change of Atrophy Level } \\
\text { After treatment (n) }\end{array}$} & \multirow[t]{2}{*}{$\begin{array}{l}\text { Effective } \\
\text { Rate[\%[ }\end{array}$} \\
\hline & & & $\begin{array}{l}\text { Non- } \\
\text { Gastritis }\end{array}$ & Mild & Moderate & Severe & $\begin{array}{l}3 \\
\text { Levels }\end{array}$ & 2 Levels & 1 Level & $\begin{array}{l}0 \\
\text { Level }\end{array}$ & \\
\hline \multirow[t]{2}{*}{ WFC } & Untreated & 30 & 0 & 15 & 11 & 4 & \multirow[t]{2}{*}{1} & \multirow[t]{2}{*}{5} & \multirow[t]{2}{*}{18} & \multirow[t]{2}{*}{6} & \multirow[t]{2}{*}{$80^{\star *}$} \\
\hline & Treated & 30 & 14 & 14 & 2 & 0 & & & & & \\
\hline \multirow[t]{2}{*}{ Con } & Untreated & 30 & 0 & 16 & 11 & 3 & \multirow[t]{2}{*}{0} & \multirow[t]{2}{*}{2} & \multirow[t]{2}{*}{5} & \multirow[t]{2}{*}{23} & \multirow[t]{2}{*}{23.33} \\
\hline & Treated & 30 & 4 & 12 & 12 & 2 & & & & & \\
\hline
\end{tabular}

WFC, Weifuchan Group; Con, Control Group; **, $P<0.01$, compared with Con.

Table \. Comparison of Gastric Mucosal Intestinal Metaplasia Level

\begin{tabular}{|c|c|c|c|c|c|c|c|c|c|c|c|}
\hline \multirow[t]{2}{*}{ Group } & & \multirow[t]{2}{*}{$n$} & \multicolumn{4}{|c|}{ Intestinal Metaplasia Level ( $n$ ) } & \multicolumn{4}{|c|}{$\begin{array}{l}\text { Change of Intestinal Metaplasia } \\
\text { Level After treatment }(n)\end{array}$} & \multirow[t]{2}{*}{$\begin{array}{l}\text { Effective } \\
\text { Rate[\%] }\end{array}$} \\
\hline & & & $\begin{array}{l}\text { Non- } \\
\text { Gastritis }\end{array}$ & Mild & Moderate & Severe & $\begin{array}{l}3 \\
\text { Levels }\end{array}$ & 2 Levels & 1 Level & $\begin{array}{l}0 \\
\text { Level }\end{array}$ & \\
\hline \multirow[t]{2}{*}{ WFC } & Untreated & 30 & 1 & 16 & 10 & 3 & \multirow[t]{2}{*}{2} & \multirow[t]{2}{*}{2} & \multirow[t]{2}{*}{18} & \multirow[t]{2}{*}{8} & \multirow[t]{2}{*}{$73.33^{\star \star}$} \\
\hline & Treated & 30 & 14 & 15 & 1 & 0 & & & & & \\
\hline \multirow[t]{2}{*}{ Con } & Untreated & 30 & 1 & 17 & 10 & 2 & \multirow[t]{2}{*}{0} & \multirow[t]{2}{*}{1} & \multirow[t]{2}{*}{7} & \multirow[t]{2}{*}{22} & \multirow[t]{2}{*}{26.67} \\
\hline & Treated & 30 & 5 & 10 & 14 & 1 & & & & & \\
\hline
\end{tabular}

WFC, Weifuchan Group; Con, Control Group; **, P<0.01, compared with Con.

Table \. Comparison of Clinical Symptom Cumulative Score

\begin{tabular}{|llrrlll|}
\hline Group & $\boldsymbol{n}$ & Untreated & Treated & Effective Rate(\%) & t & $\boldsymbol{P}$ \\
WFC & 30 & $15.32 \pm 5.71$ & $6.27 \pm 2.19$ & 86.67 & 23.231 & $<0.01$ \\
Con & 30 & $15.92 \pm 5.43$ & $13.69 \pm 4.12$ & 23.33 & & \\
\hline
\end{tabular}

WFC, Weifuchan Group; Con, Control Group;

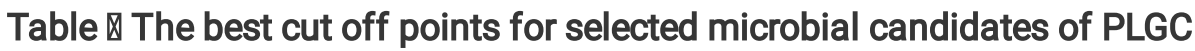




\begin{tabular}{|llllllll|}
\hline OUT & Area & $\begin{array}{l}\text { Lower } \\
\text { Bound }\end{array}$ & $\begin{array}{l}\text { Upper } \\
\text { Bound }\end{array}$ & $\begin{array}{l}\text { Cut off } \\
\text { Value }\end{array}$ & Sensitivity & Specificity & $\begin{array}{c}\boldsymbol{p} \text { Value } \\
\text { Vacteroides }\end{array}$ \\
\hline Lachnospiraceae;Other & 0.66 & 0.44 & 0.88 & 3.494 & 0.43 & 1 & 0.178 \\
\hline Faecalibacterium & 0.58 & 0.33 & 0.82 & 89550.33 & 0.93 & 0.2 & 0.520 \\
\hline Lachnoclostridium & 0.53 & 0.29 & 0.77 & 96980.75 & 0.21 & 0.9 & 0.815 \\
\hline Parabacteroides & 0.55 & 0.31 & 0.79 & 19030.36 & 0.71 & 0.5 & 0.682 \\
\hline Streptococcus & 0.77 & 0.57 & 0.97 & 18354.22 & 0.79 & 0.8 & 0.026 \\
\hline Escherichia-Shigella & 0.58 & 0.34 & 0.81 & 4847.02 & 0.57 & 0.8 & 0.520 \\
\hline $\begin{array}{l}\text { Lachnospiraceae NK4A136 } \\
\text { group }\end{array}$ & 0.55 & 0.31 & 0.79 & 3969.2 & 0.43 & 0.8 & 0.682 \\
\hline
\end{tabular}

Figures 


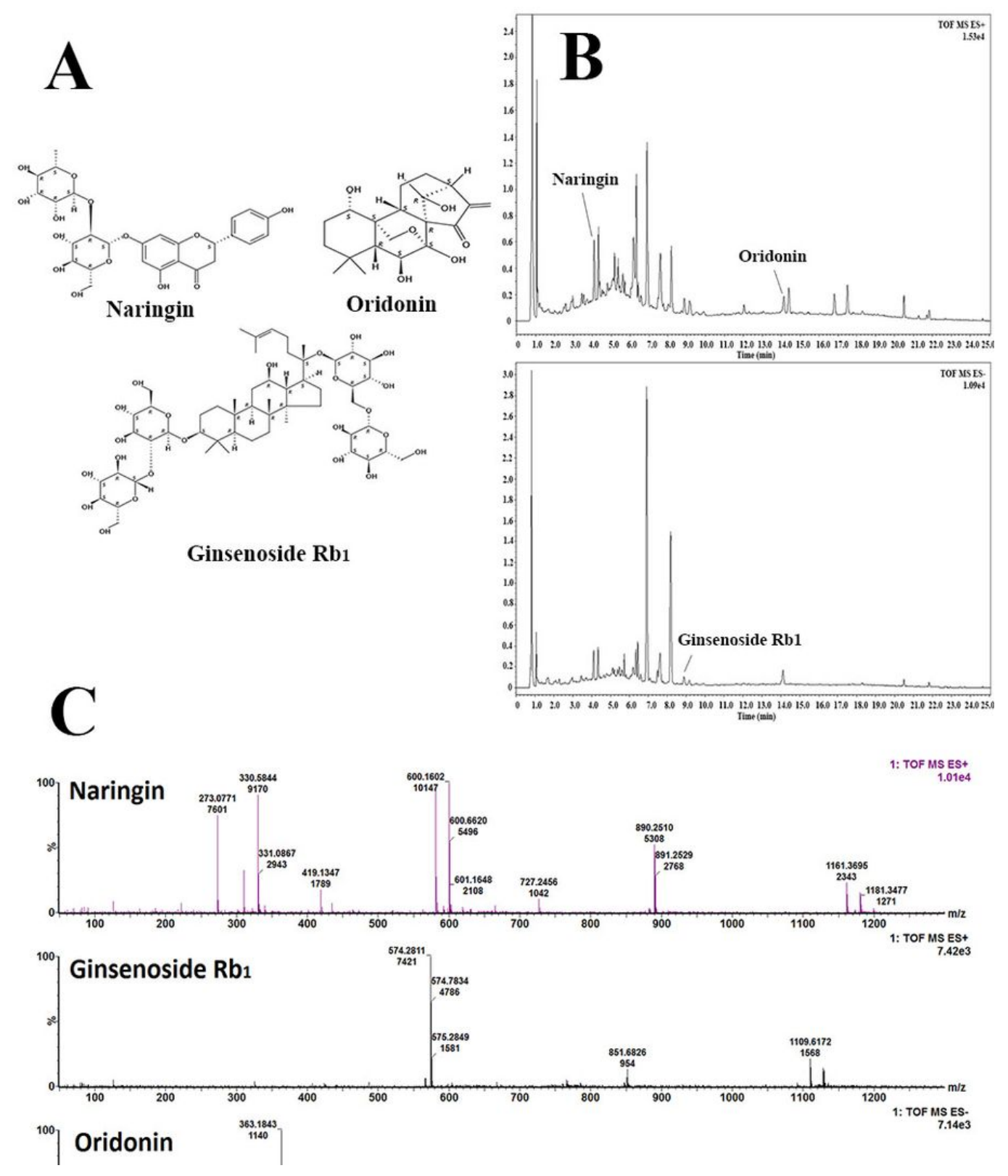

\section{Figure 1}

The naringin, ginsenoside Rb1, and oridonin contents in WFC. (A)The molecular structure of naringin, ginsenoside Rb1, and oridonin. (B) TOF MS chromatogram of WFC in positive and negative model. The peaks for naringin, ginsenoside $\mathrm{Rb} 1$, and oridonin are indicated by a line. (C) Mass spectra of naringin (upper), ginsenoside Rb 1 (middle), and oridonin (lower). The mass-to-charge $(\mathrm{m} / \mathrm{z})$ ratios of all detected peaks are shown above each peak. 


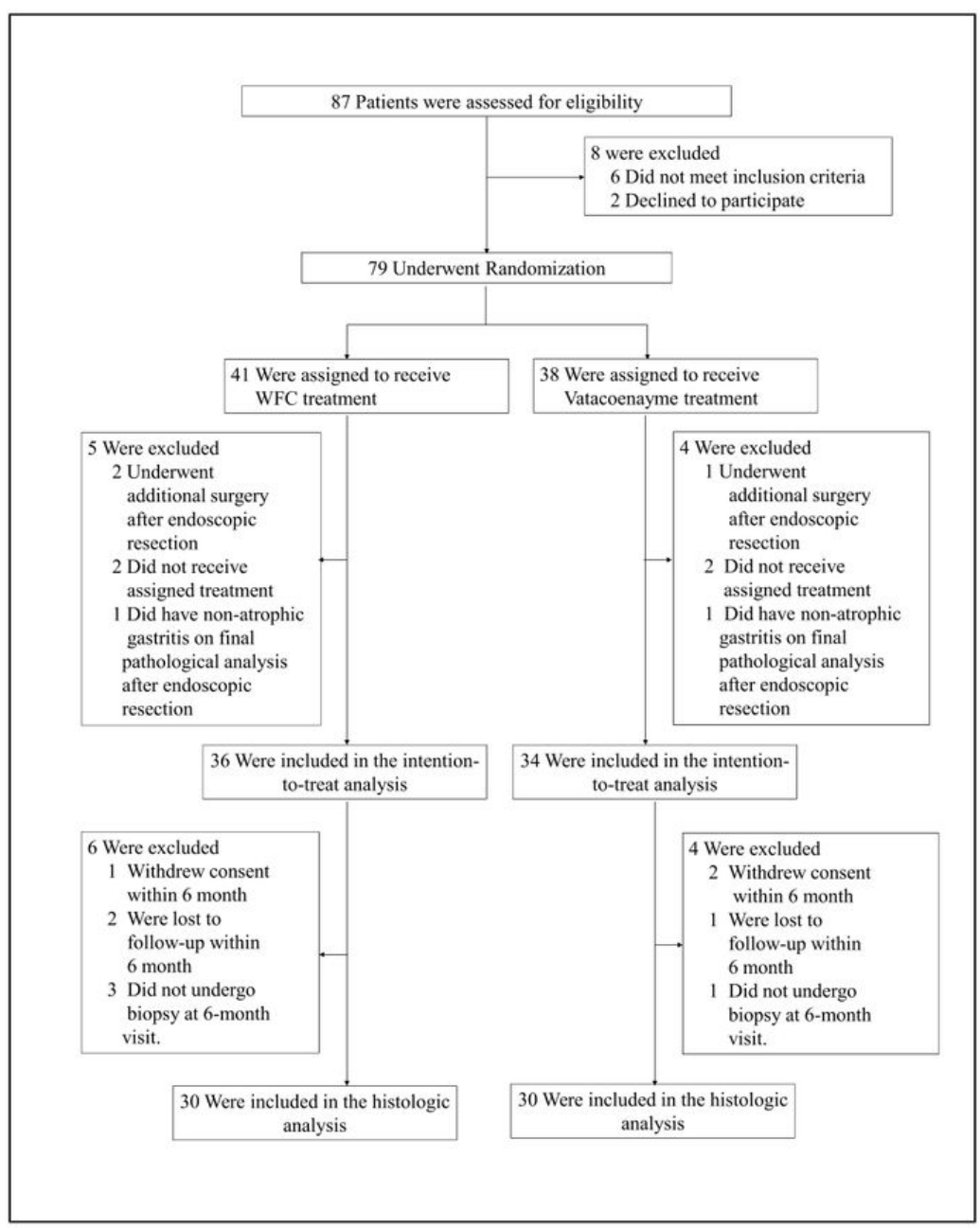

\section{Figure 2}

Enrollment, Randomization, and Follow-up. The improvement from baseline in the grade of histopathology detected on gastroscopy at the 6-month follow-up was evaluated in the intention-to treat population. Only patients with gastric tissue specimens obtained at the 6-month follow-up were included in the histologic analysis. 
A

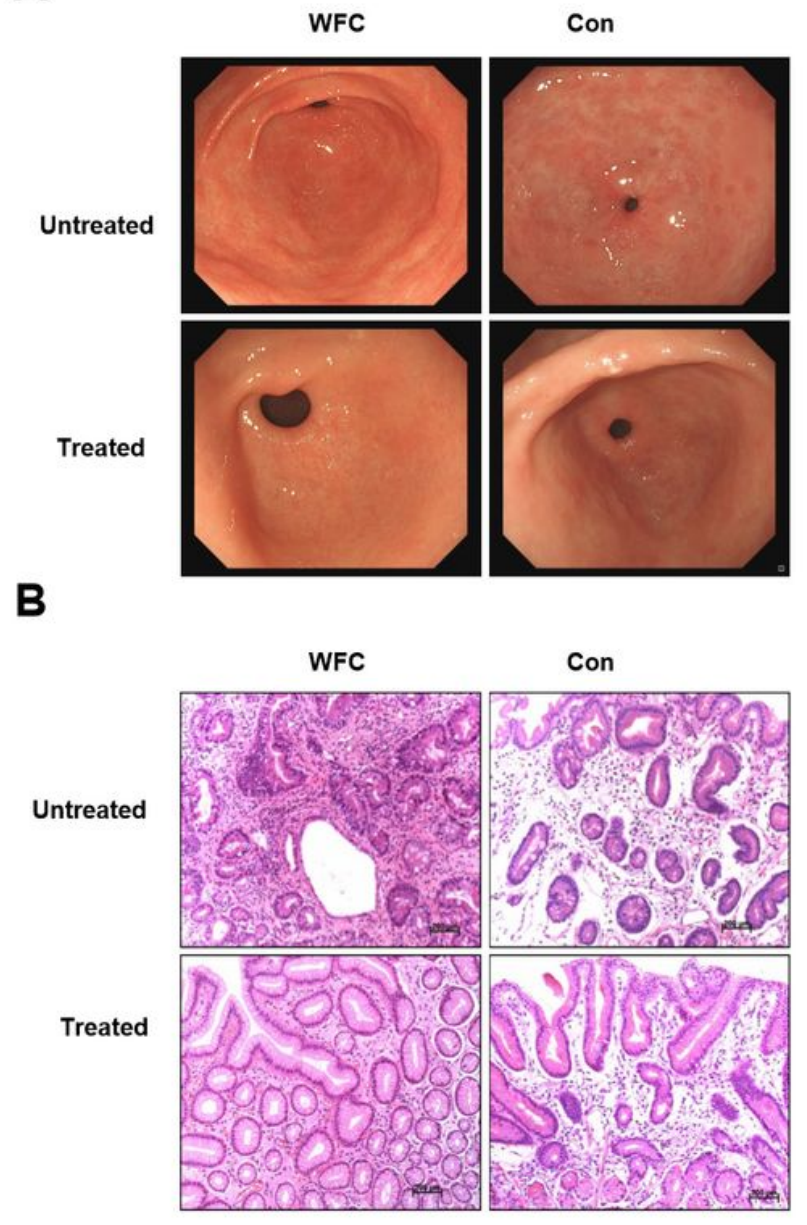

C
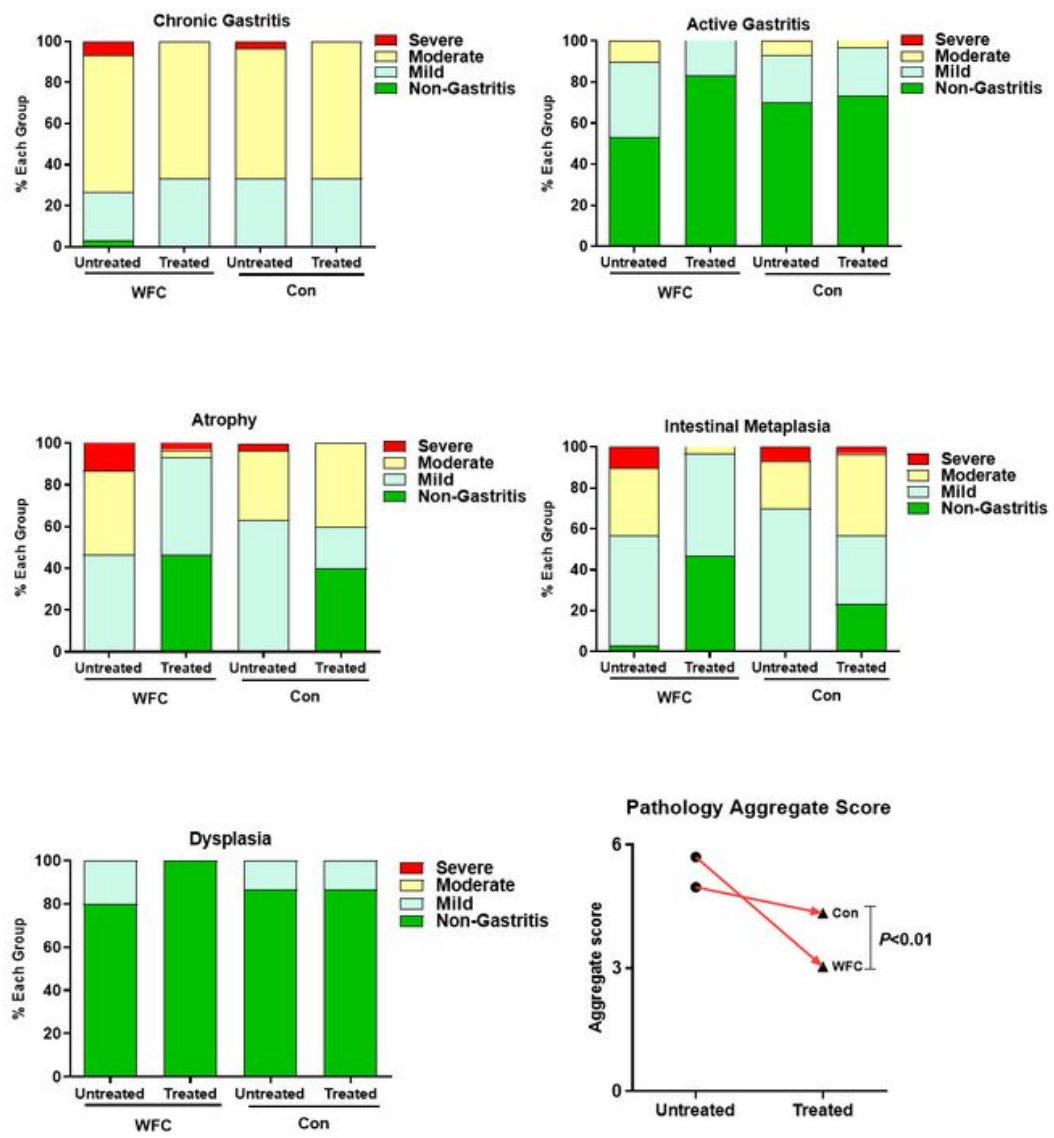

\section{Figure 3}

Histopathological Variation. (A) Endoscope variation. (B) Pathological staining in HE. (C) Pathological evaluation. WFC, Weifuchan Group; Con, Control Group; 
Phyla

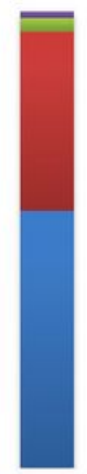

Health
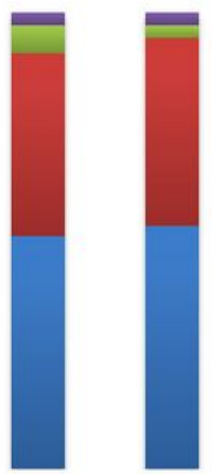

WFCB WFCA

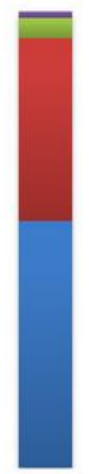

ConB

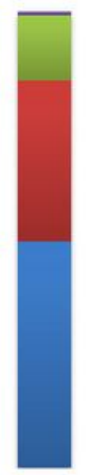

ConA nother

= Proteobacteria

- Bacteroidetes

airmicutes

\section{Genera}

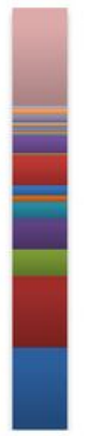

Health

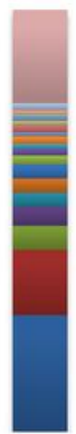

WFCB WFCA

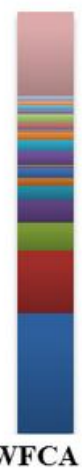

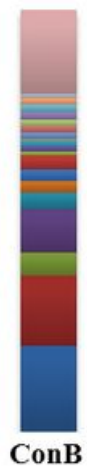

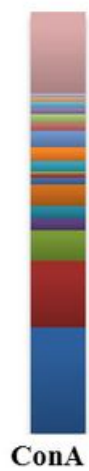

Class
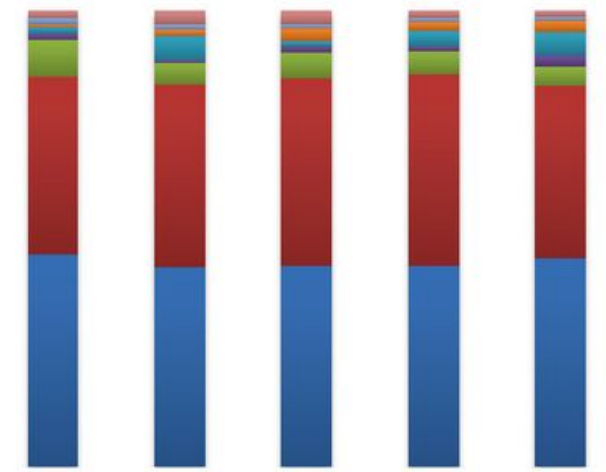

w other

Erysipelotrichia

vacilli

= Gammapr oteobacteria

- Betaproteobacteria

Negativicutes

n Bacteroidia

- Clostridia

Health WFCB WFCA ConB ConA

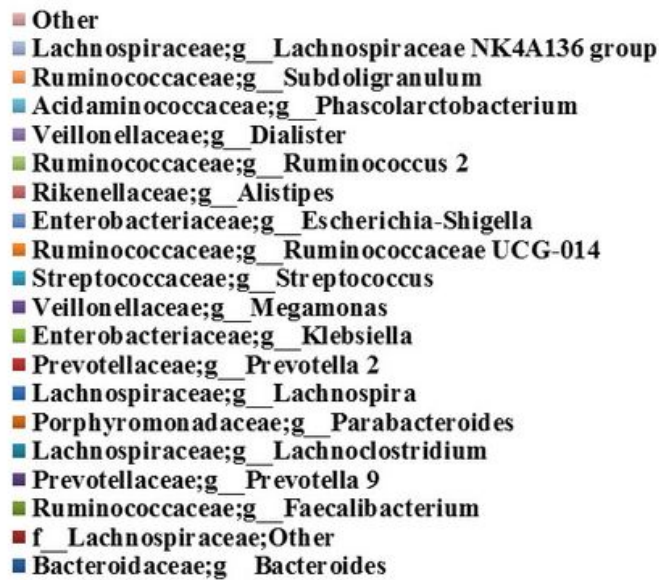

Figure 4

Microbial profiles $₫$ mean relative proportion $₫$ of most abundant $₫>1 \% \bigotimes$ phyla, class and genera by comparison for groups. Health: healthy volunteers; WFCB: PLGC patients before treatment with WFC; WFCA: PLGC patients after treatment with WFC; ConB: PLGC patients before treatment with control drug; ConA: PLGC patients after treatment with control drug. 

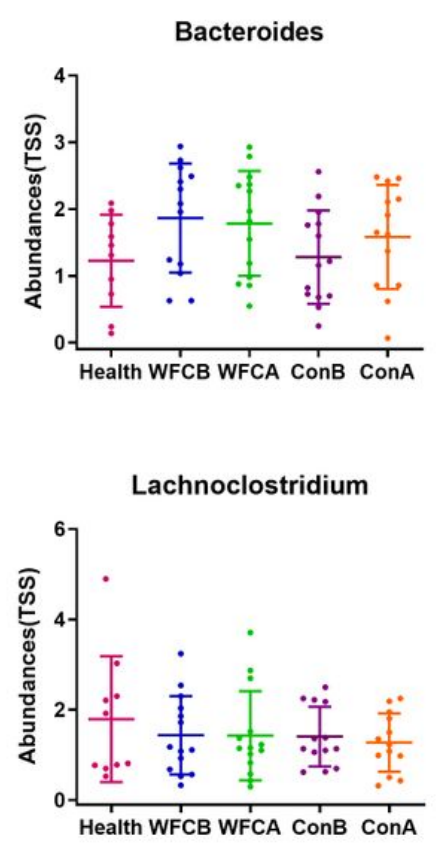

Streptococcus

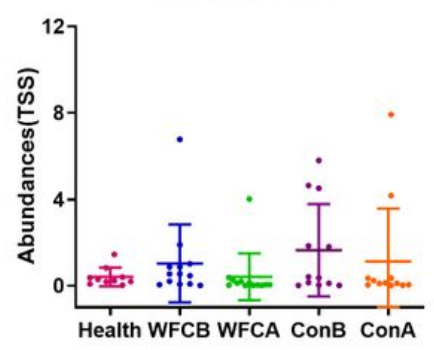

Lachnospiraceae NK4A136 group

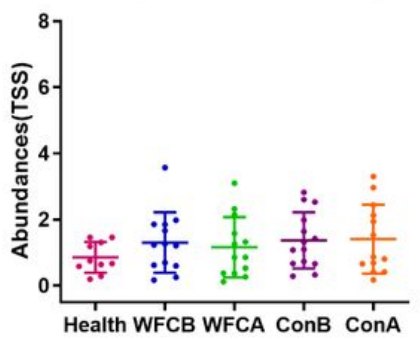

Faecalibacterium

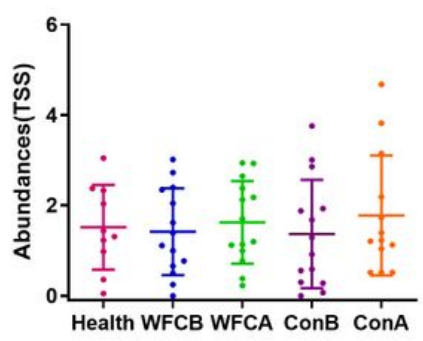

Parabacteroides

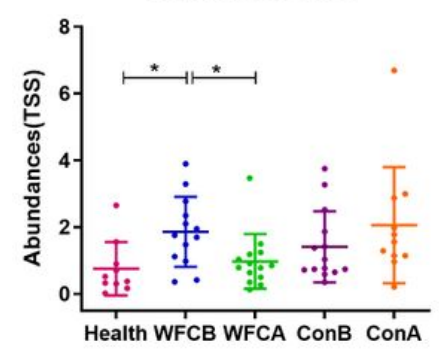

Escherichia-Shigella

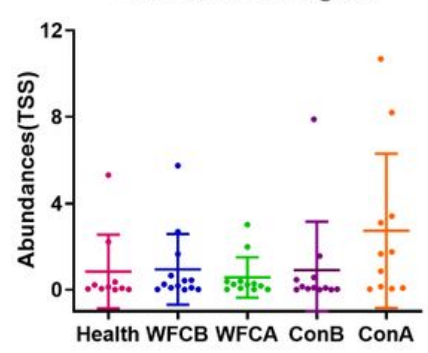

Lachnospiraceae;Other

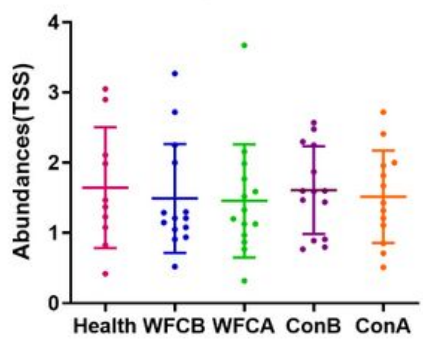

\section{Figure 5}

Comparisons for bacterial abundance. Bacterial relative abundance differences were observed for 8 core genera in each group. TSS: Total -sum normalization. ${ }^{*}, \mathrm{p}<0.05$. 
ROC Curve

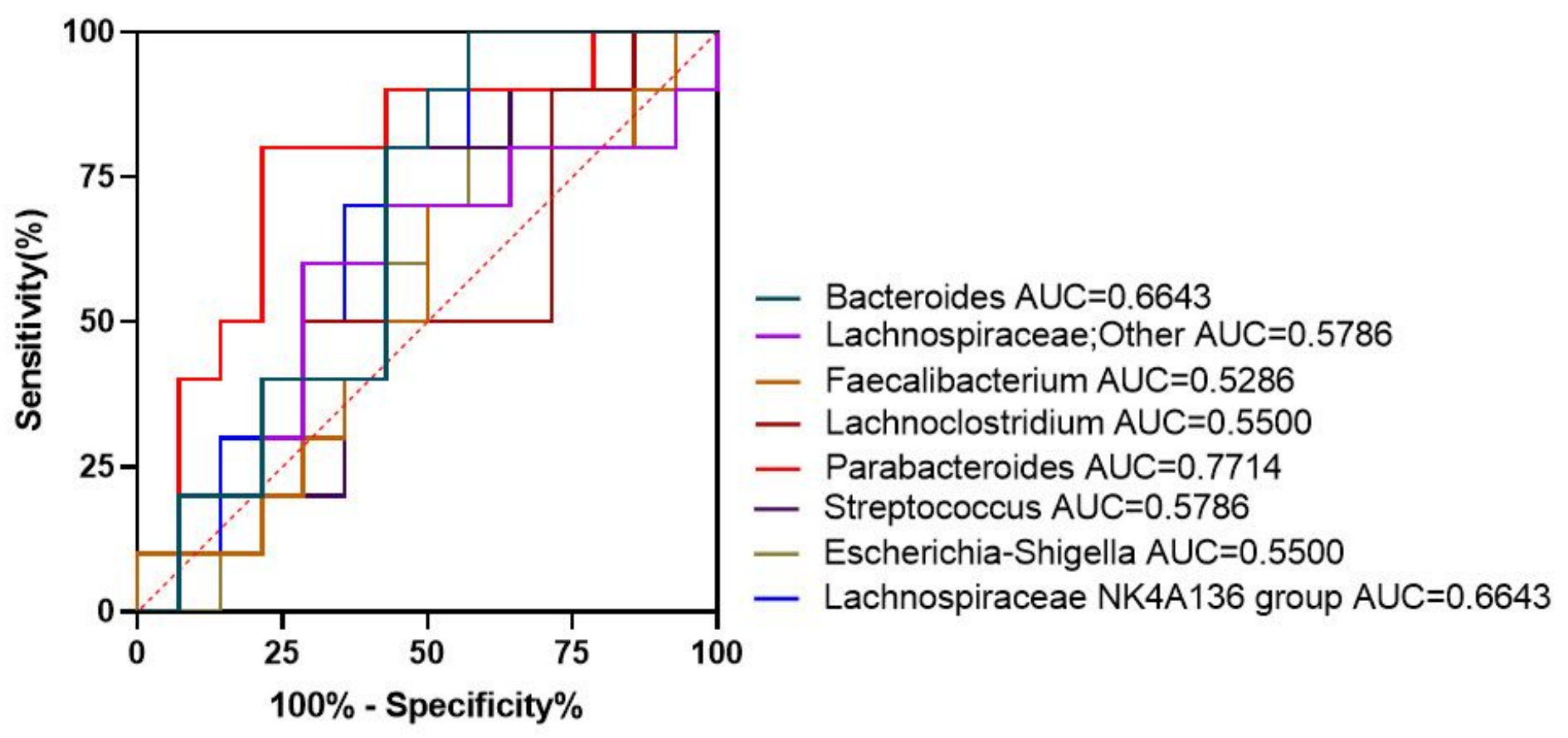

Figure 6

Receiver operating curve analysis for selected micro biomarker of PLGC. 8 sleceted microbial biomarkkers of PLGC were tested by ROC analysis. 